\title{
Intercostal artery incorporation to prevent spinal cord ischemia during total endovascular thoracoabdominal aortic repair
}

\author{
Anastasia Plotkin, MD, ${ }^{a}$ Sukgu M. Han, MD, MS, ${ }^{a}$ Miguel F. Manzur, MD, ${ }^{a}$ Mark J. Cunningham, MD, ${ }^{b}$ \\ Fernando Fleischman, MD, ${ }^{\mathrm{b}}$ and Gregory A. Magee, MD, MSc, FACS, ${ }^{\mathrm{a}}$ Los Angeles, Calif
}

\footnotetext{
From the Divisions of ${ }^{\mathrm{a}}$ Vascular Surgery and Endovascular Therapy and ${ }^{\mathrm{b}}$ Cardiothoracic Surgery, Department of Surgery, Keck School of Medicine of USC, University of Southern California, Los Angeles, Calif.

Disclosures: Drs Han and Fleishman have received consulting fees from Cook Medical and WL Gore \& Associates. Drs Cunningham and Magee have received consulting fees from WL Gore \& Associates. All other authors reported no conflicts of interest.

The Journal policy requires editors and reviewers to disclose conflicts of interest and to decline handling or reviewing manuscripts for which they may have a conflict of interest. The editors and reviewers of this article have no conflicts of interest.

Accepted for the American Association for Thoracic Surgery Aortic Symposium 2020.

Received for publication Jan 13, 2021; accepted for publication Jan 19, 2021; available ahead of print Jan 28, 2021 .

Address for reprints: Gregory A. Magee, MD, MSc, FACS, Division of Vascular Surgery and Endovascular Therapy, Department of Surgery, University of Southern California, 1520 San Pablo St, Suite 4300, Los Angeles, CA 90033 (E-mail: gregory.magee@med.usc.edu).

JTCVS Techniques 2021;6:32-6

2666-2507

Copyright (C) 2021 The Authors. Published by Elsevier Inc. on behalf of The American Association for Thoracic Surgery. This is an open access article under the CC BY-NC-ND license (http://creativecommons.org/licenses/bync-nd/4.0/).

https://doi.org/10.1016/j.xjtc.2021.01.020
}

Video clip is available online.

Spinal cord ischemia (SCI) is a devastating complication of aortic repair with multiple strategies developed specifically to prevent and mitigate its occurrence by preserving spinal cord perfusion. This includes permissive hypertension, limiting endovascular aortic coverage, reattachment of intercostal arteries during open repair, steroid infusion, and spinal fluid drainage. ${ }^{1-4}$

Intercostal artery preservation techniques aimed to reduce spinal cord ischemia during open thoracoabdominal aortic aneurysm (TAAA) repairs have been well described. ${ }^{5}$ In contrast, coverage of intercostal and lumbar arteries has been considered a necessity during endovascular TAAA repairs with most of the SCI prevention measures focused on indirect augmentation of spinal cord perfusion pressures. These measures include placement of a spinal fluid drain, augmentation of mean arterial pressure, and liberal transfusion threshold. However, with advancements in complex endovascular aortic repair techniques using fenestrated and branched grafts, intercostal preservation may be possible in select patients.

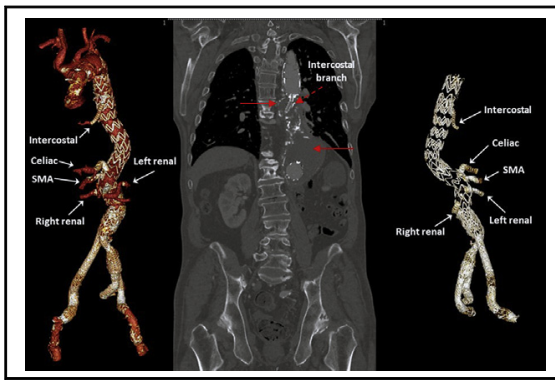

Completion CT pseudoaneurysm thrombosis (solid arrows) and $3 \mathrm{D}$ recon aorta and graft.

CENTRAL MESSAGE

Intercostal artery incorporation during endovascular repair of the thoracoabdominal aorta is feasible and can further decrease the rate of spinal cord ischemia.

See Commentary on page 37.
We describe a case of symptomatic intercostal Carrel patch pseudoaneurysms after open thoracoabdominal aortic repair that was successfully treated with staged total endovascular repair with preservation of a dominant intercostal artery. Patient consent was obtained for publication of this report and institutional review board approval was exempt at our institution. The procedures described represent offlabel use of devices using techniques that have been previously described. ${ }^{6}$

\section{CASE DESCRIPTION}

A 71-year-old man with a prior open total arch repair with reimplantation of all 3 arch branches 16 years prior followed by open Crawford extent II TAAA repair with intercostal and visceral (celiac and superior mesenteric artery [SMA]) Carrel patches and branch grafts for the renal arteries 1 year later presented to us with 2 episodes of large volume hemoptysis. He was found to have large pseudoaneurysms of his intercostal and visceral artery Carrel patches, measuring $5.3 \times 4.1 \mathrm{~cm}$ and $9.4 \times 8.1 \mathrm{~cm}$, respectively. Computed tomography angiography (CTA) did not 
clearly delineate an aortobronchial fistula; however, given the history of multiple episodes of large volume hematemesis, blood in the left lower lobe on bronchoscopy, and inflammatory changes in the lung adjacent to the pseudoaneurysms, aortobronchial fistula was the presumed diagnosis (Figure 1). Given his age and extensive previous open repairs, he was deemed high risk for repeat open repair. Imaging also demonstrated a large intercostal artery at the level of $\mathrm{T} 8$ and therefore discussions of repair centered around its preservation to prevent spinal cord ischemia. Staged endovascular repair was planned with the first procedure to repair the intercostal patch pseudoaneurysm with incorporation of the large intercostal artery using a branched thoracic endograft followed by a 4-vessel fenestrated endovascular aortic aneurysm repair with bifurcated endovascular aortic aneurysm repair and left iliac branch endograft to treat his visceral patch pseudoaneurysm as well as a $3.5-\mathrm{cm}$ left common iliac artery aneurysm.

A Zenith Alpha $38 \times 34 \times 167 \mathrm{~mm}$ thoracic endograft (Cook Medical, Bloomington, Ind) was modified with a single caudally directed side branch and a precannulated wire as previously described. ${ }^{6} \mathrm{Next}$, left brachial to right femoral through-wire access was obtained for placement of the precannulated wire. An aortogram was performed to demonstrate the location of the intercostal patch pseudoaneurysm and the large intercostal artery (Figure 2). The endograft was advanced and partially deployed with its branch oriented toward the intercostal artery. The intercostal artery was then cannulated from the left brachial access and stented with a $6 \times 79 \mathrm{~mm}$ VBX (WL Gore \& Associates, Flagstaff, Ariz). Completion angiogram verified complete seal of the proximal patch pseudoaneurysm, preservation of the dominant intercostal artery, and the distal large pseudoaneurysm (Figure 2, C).

Subsequently, he underwent repair of his remaining $9.4 \mathrm{~cm}$ visceral patch pseudoaneurysm using a 4-vessel fenestrated graft constructed using a Zenith Alpha $34 \times 34 \times 161 \mathrm{~mm}$ with branches for the celiac and superior mesenteric artery and fenestrations for the renal arteries. The modification steps have been previously published. ${ }^{6}$ After bilateral femoral access was established, an angiogram with 3-dimensional fusion was performed to appropriately mark the location of visceral branches. After deployment of the branched-fenestrated endograft, each visceral branch was individually selected and stented. Finally, a $35 \times 14 \times 180 \mathrm{~mm}$ Excluder with left iliac branch graft (WL Gore \& Associates) was used to repair bilateral

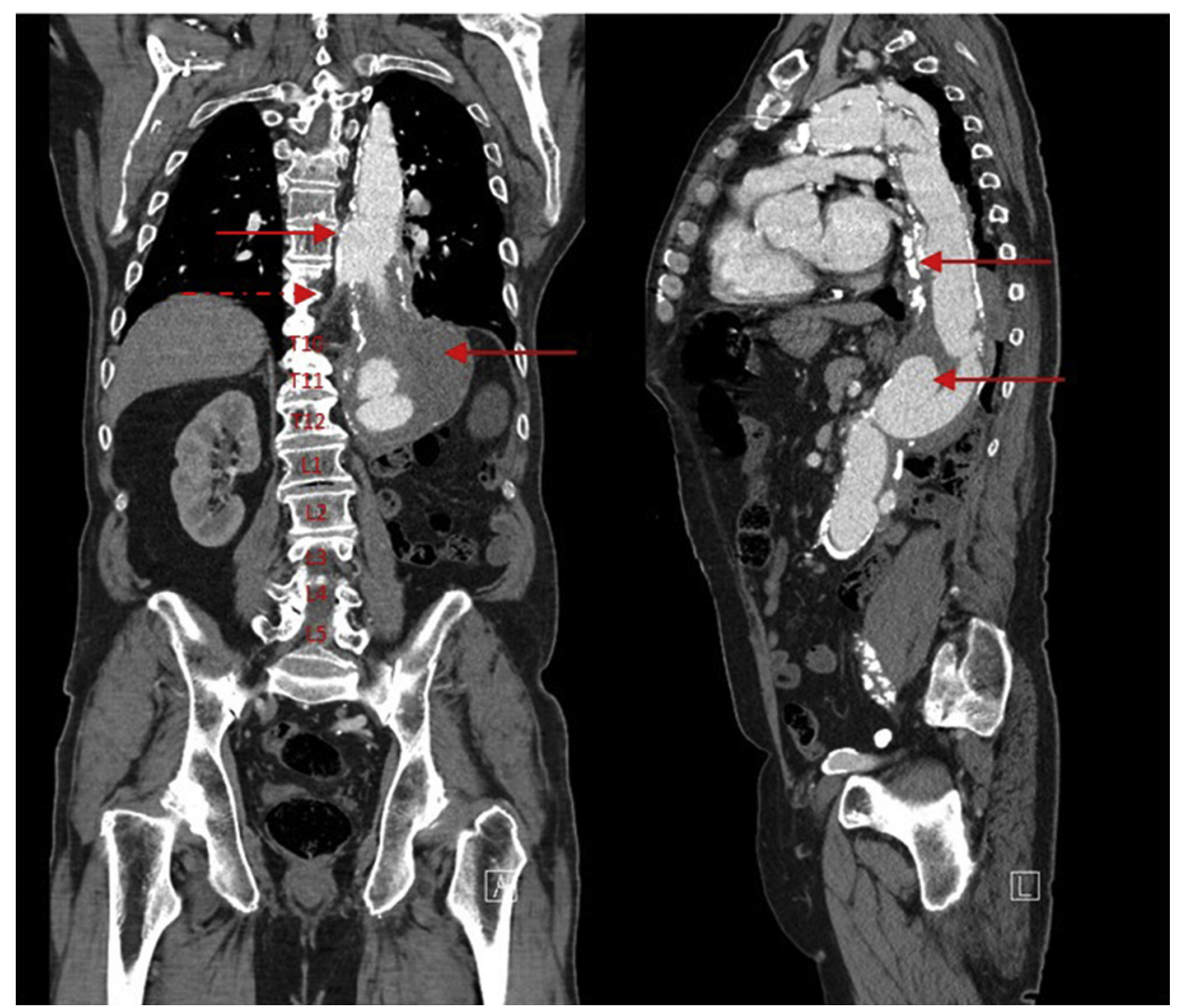

FIGURE 1. Preoperative imaging demonstrating 2 pseudoaneurysms (solid arrows) at the Carrel patches from previous open aortic repair and a large intercostal artery (dashed arrow). 


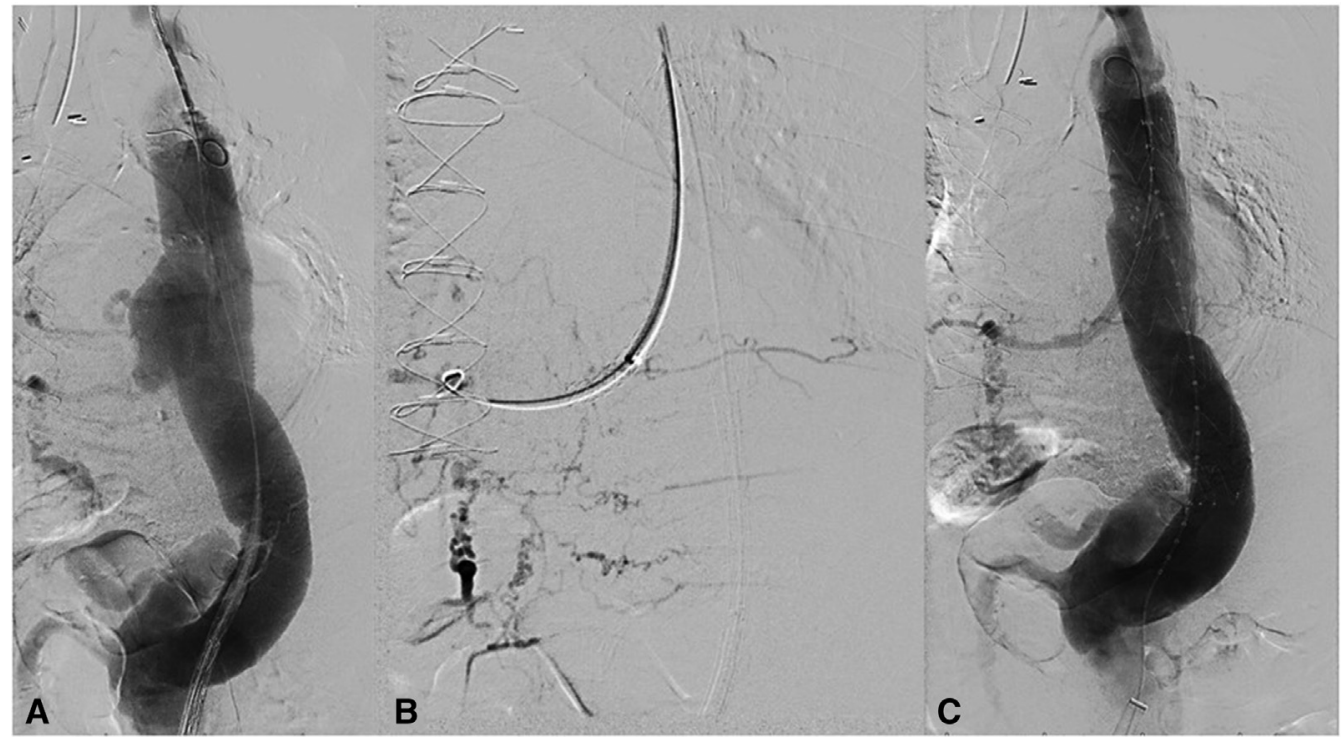

FIGURE 2. A, Intercostal angiogram during endovascular incorporation of the intercostal artery, demonstrates both Carrel patch pseudoaneurysms that resulted after open thoracoabdominal aneurysm repair. B, Fluoroscopic images show cannulation of the large intercostal artery, stent deployment into the intercostal artery, and contrast within associated collateral perfusion. C, After deployment of the endograft and intercostal stent, completion angiogram demonstrates appropriate thoracic endovascular aortic graft and intercostal stent apposition.

iliac aneurysms (Figure 3). His postoperative course was uneventful and he was discharged home without complications. He was placed on dual antiplatelet therapy after the procedure to help aid patency of the intercostal branch. Postoperative CTA demonstrated complete thrombosis of both pseudoaneurysms, preservation of flow into all stented branches, and no endoleak (Figure 4). Now 1 year postoperatively, he has had no further hemoptysis, continues to have normal functional status, and remains on lifelong suppressive antibiotics. Surveillance CTA demonstrates patency of all the branches, including of the intercostal artery (Video 1). Due to preservation of his intercostal artery, all of the endovascular repairs were performed without spinal fluid drainage or neuromonitoring.

\section{DISCUSSION}

Although intercostal-lumbar arteries can be readily identified on preoperative or intraoperative imaging, the

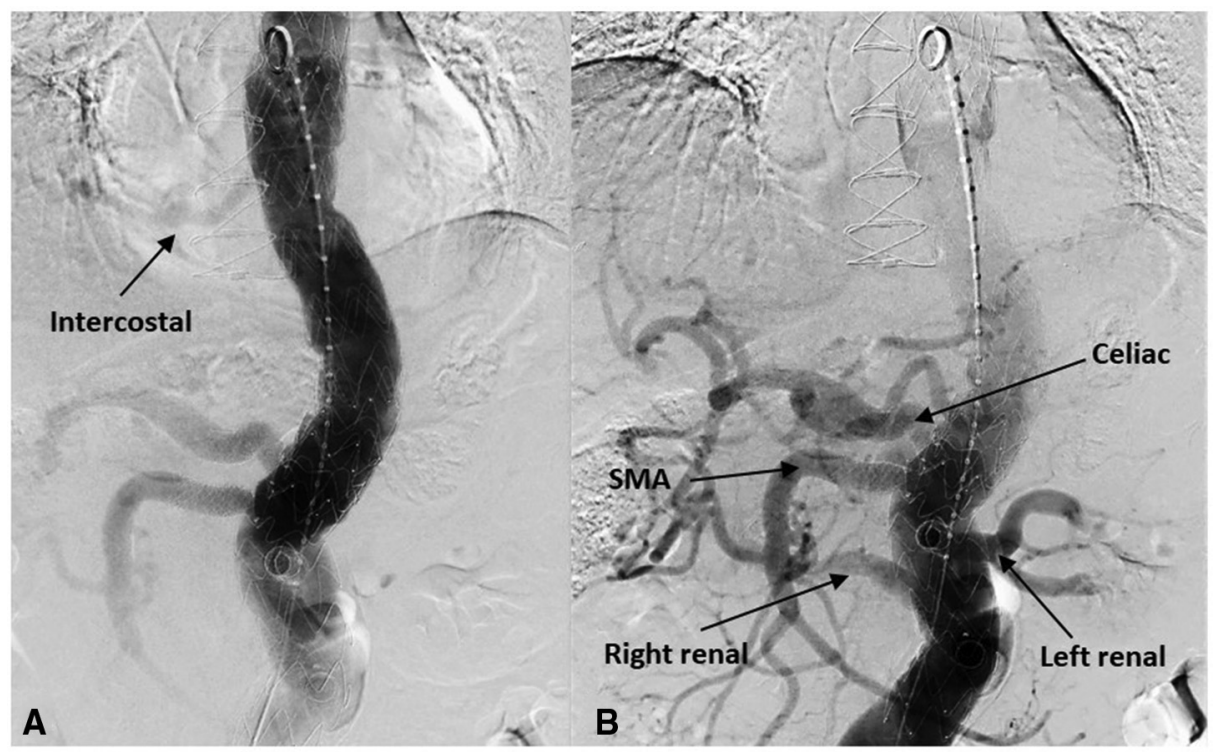

FIGURE 3. After thoracic endograft repair with intercostal artery incorporation (A), the subsequent stage of abdominal aortic repair was performed and completion angiogram demonstrates appropriate filling of all visceral branches after this fenestrated endovascular repair (B). SMA, Superior mesenteric artery. 


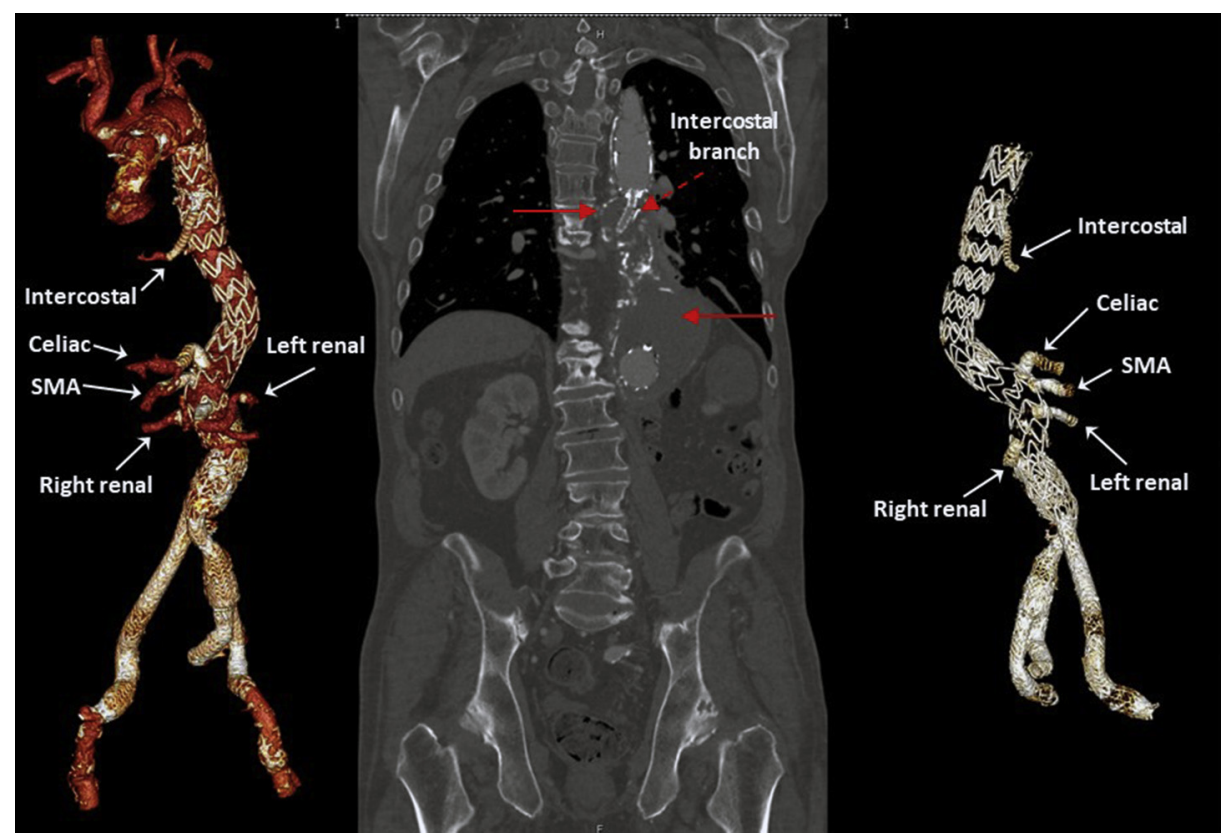

FIGURE 4. At follow-up after thoracic aortic repair of Carrel patch pseudoaneurysms with large intercostal artery incorporation and fenestrated endovascular abdominal aortic repair, completion computed tomography demonstrates pseudoaneurysm thrombosis (solid arrows). Also shown is the 3-dimensional reconstruction of aorta after endograft repair and the isolated graft structure. SMA, Superior mesenteric artery.

individual vessels are usually small and not amenable to endovascular stenting. Hence, little to no data exist on their incorporation during endovascular aortic repair. ${ }^{1,7}$ Efforts are instead aimed at limiting the extent of aortic repair to avoid coverage of numerous intercostal-lumbar arteries during a single operation. Therefore, most adopt a staged repair approach to extensive thoracoabdominal pathologies. ${ }^{8}$ In addition to the adjunctive measures to indirectly augment spinal cord perfusion such as cerebrospinal fluid drain and mean arterial pressure augmentation that are widely performed, newer approaches to spinal cord preconditioning by selective intercostal and lumbar embolization have been performed and are currently undergoing a randomized trial in Europe. ${ }^{1-3,9-11}$ However, select patients with a

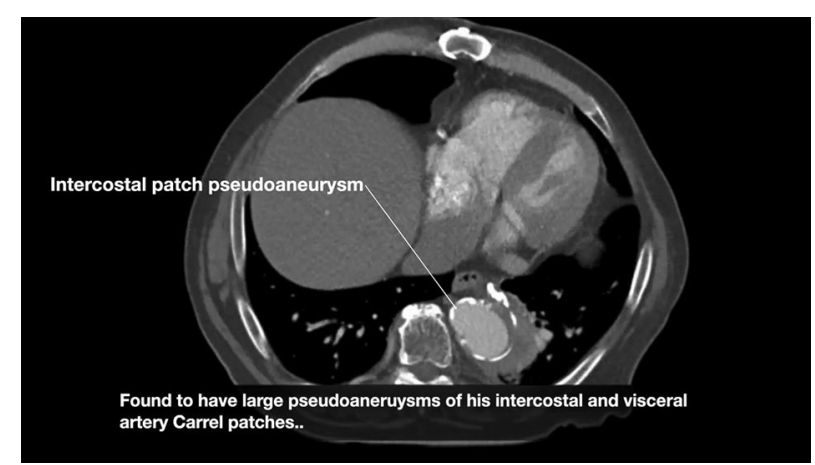

VIDEO 1. Intercostal artery incorporation to prevent spinal cord ischemia during total endovascular thoracoabdominal aortic repair. Video available at: https://www.jtcvs.org/article/S2666-2507(21)00087-0/fulltext. large dominant intercostal artery may be candidates for intercostal artery incorporation during endovascular aortic repairs. In our case, the patient underwent reimplantation of a large intercostal artery at the level of T8 as a Carrel patch during previous open Extent II thoracoabdominal aneurysm repair. Because this intercostal artery was $5 \mathrm{~mm}$ in diameter and collateralized to several other intercostals, we decided to preserve it with a branched graft.

Intercostal and lumbar artery preservation can be more readily performed during open aortic repair. Although its technique and technical feasibility have been well described by high-volume centers of excellence, intercostal preservation during open TAAA repair has not been widely adopted and its benefit in spinal cord protection is not easily duplicated. ${ }^{5}$ This may be due to transient loss of spinal perfusion pressure during open intercostal reimplantation or bypass. On the contrary, during endovascular incorporation, there is little to no interruption of flow to the target intercostal artery. It is important to note that Carrel patch pseudoaneurysms are commonly seen after open thoracoabdominal repair, especially in patients with connective tissue disorders; therefore, we recommend routine surveillance in these patients. ${ }^{12}$

Requirements for successful outcomes of intercostal preservation include the target vessel size and the surgeon's familiarity with advanced endovascular techniques. Currently, the smallest available covered stents are $5 \mathrm{~mm}$ in diameter. Therefore, vessels smaller than $5 \mathrm{~mm}$ in diameter are difficult to incorporate into endovascular repair and likely result in lower patency. Preservation of intercostal 
arteries during endovascular repair has the potential to decrease the risk of spinal cord ischemia, but further studies are necessary to determine the efficacy of this technique.

We believe a multimodal approach is ideal for optimal SCI risk mitigation during complex endovascular aortic repair. This may include spinal fluid drainage, limiting extent of aortic endovascular coverage, permissive hypertension, and intercostal/lumbar artery preservation if possible. After review of our data, we have shifted away from routinely performing prophylactic lumbar drainage for thoracic and thoracoabdominal endovascular repair. Instead, we place prophylactic lumbar drains in the most high-risk elective patients with concomitant intraoperative neuromonitoring and placing immediate lumbar drains therapeutically when needed. ${ }^{13}$ Mean arterial pressure augmentation is performed in all patients. A similar change in practice has also occurred at other high-volume centers. ${ }^{14,15}$

\section{CONCLUSIONS}

Intercostal artery preservation is feasible and may be a valuable adjunct in endovascular aortic repair, especially in cases where a large, dominant intercostal artery is present. Use of this technique may further reduce the rate SCI following endovascular repair of thoracoabdominal aortic aneurysms.

\section{References}

1. Matsuda H, Ogino H, Fukuda T, Iritani O, Sato S, Iba Y, et al. Multidisciplinary approach to prevent spinal cord ischemia after thoracic endovascular aneurysm repair for distal descending aorta. Ann Thorac Surg. 2010;90:561-5.

2. Scali ST, Kim M, Kubilis P, Feezor RJ, Giles KA, Miller B, et al. Implementation of a bundled protocol significantly reduces risk of spinal cord ischemia after branched or fenestrated endovascular aortic repair. J Vasc Surg. 2018;67: 409-23.e4.
3. Drinkwater SL, Goebells A, Haydar A, Bourke P, Brown L, Hamady M, et al. Incidence of spinal cord ischaemia following thoracic and thoracoabdominal aortic endovascular intervention. Eur J Vasc Endovasc Surg. 2010; 40:729-35.

4. Afifi RO, Sandhu HK, Zaidi ST, Trinh E, Tanaka A, Miller CC III, et al. Intercostal artery management in thoracoabdominal aortic surgery: to reattach or not to reattach? J Thorac Cardiovasc Surg. 2018;155:1372-8.e1.

5. Safi HJ, Miller CC III, Carr C, Iliopoulos DC, Dorsay DA, Baldwin JC. Importance of intercostal artery reattachment during thoracoabdominal aortic aneurysm repair. J Vasc Surg. 1998;27:58-66.

6. Han SM, Tenorio ER, Mirza AK, Zhang L, Weiss S, Oderich GS. Low-profile Zenith Alpha thoracic stent graft modification using preloaded wires for urgent repair of thoracoabdominal and pararenal abdominal aortic aneurysms. Ann Vasc Surg. 2020;67:14-25.

7. Koutouzi G, Sandstrom C, Skoog P, Roos H, Falkenberg M. 3D image fusion to localise intercostal arteries during TEVAR. EJVES Short Rep. 2017;35:7-10.

8. O'Callaghan A, Mastracci TM, Eagleton MJ. Staged endovascular repair of thoracoabdominal aortic aneurysms limits incidence and severity of spinal cord ischemia. J Vasc Surg. 2015;61:347-54.e1.

9. Magee GA, Yi JA, Kuwayama DP. Intercostal artery embolization to induce false lumen thrombosis in type B aortic dissection. J Vasc Surg Cases Innov Tech. 2020;6:433-7.

10. Etz CD, Debus ES, Mohr FW, Kolbel T. First-in-man endovascular preconditioning of the paraspinal collateral network by segmental artery coil embolization to prevent ischemic spinal cord injury. J Thorac Cardiovasc Surg. 2015;149: 1074-9.

11. Petroff D, Czerny M, Kolbel T, Melissano G, Lonn L, Haunschild J, et al. Paraplegia prevention in aortic aneurysm repair by thoracoabdominal staging with 'minimally invasive staged segmental artery coil embolisation' (MIS(2)ACE): trial protocol for a randomised controlled multicentre trial. BMJ Open. 2019;9: e025488.

12. Dardik A, Perler BA, Roseborough GS, Williams GM. Aneurysmal expansion of the visceral patch after thoracoabdominal aortic replacement: an argument for limiting patch size? J Vasc Surg. 2001;34:405-9.

13. Plotkin A, Han SM, Weaver FA, Rowe VL, Ziegler KR, Fleischman F, et al. Complications associated with lumbar drain placement for endovascular aortic repair. J Vasc Surg. October 11, 2020 [Epub ahead of print].

14. Weissler EH, Voigt SL, Raman V, Jawitz O, Doberne J, Anand J, et al. Permissive hypertension and collateral revascularization may allow avoidance of cerebrospinal fluid drainage in thoracic endovascular aortic repair. Ann Thorac Surg. 2020; 110:1469-74.

15. Karkkainen JM, Cirillo-Penn NC, Sen I, Tenorio ER, Mauermann WJ, Gilkey GD. Cerebrospinal fluid drainage complications during first stage and completion fenestrated-branched endovascular aortic repair. J Vasc Surg. 2020; 71:1109-18.e2. 